Revue musicale OICRM

\title{
Présentation du numéro " Mémoire musicale et résistance. Autour du Verfügbar aux Enfers de Germaine Tillion »
}

\section{Marie-Hélène Benoit-Otis et Philippe Despoix}

Volume 3, numéro 2, 2016

Mémoire musicale et résistance. Autour du Verfügbar aux Enfers de Germaine Tillion

URI : https://id.erudit.org/iderudit/1060103ar

DOI : https://doi.org/10.7202/1060103ar

Aller au sommaire du numéro

Éditeur(s)

OICRM

ISSN

2368-7061 (numérique)

Découvrir la revue

\section{Citer ce document}

Benoit-Otis, M.-H. \& Despoix, P. (2016). Présentation du numéro « Mémoire musicale et résistance. Autour du Verfügbar aux Enfers de Germaine Tillion ». Revue musicale OICRM, 3(2), i-iv. https://doi.org/10.7202/1060103ar 


\section{revue musicale oicrm}

Le site de la Revue musicale de l'Observatoire interdisciplinaire de création et recherche en musique

\section{Présentation du numéro « Mémoire musicale et résistance. Autour du Verfügbar aux Enfers de Germaine Tillion », vol. 3, $\mathrm{n}^{\circ} 2$ (2016) Marie-Hélène Benoit-Otis
et Philippe Despoix}

Les études qui composent le présent numéro explorent, sous des angles différents et complémentaires, les dimensions de résistance propre à la création artistique dans une situation extrême comme celle des camps de concentration. Les réflexions proposées ici s'articulent essentiellement autour d'un document musico-théâtral exceptionnel : Le Verfügbar aux Enfers, opérette-revue sans partition, rédigée avec l'aide de ses compagnes d'infortune par l'ethnologue française Germaine Tillion à l'automne 1944, alors qu'elle était détenue comme résistante

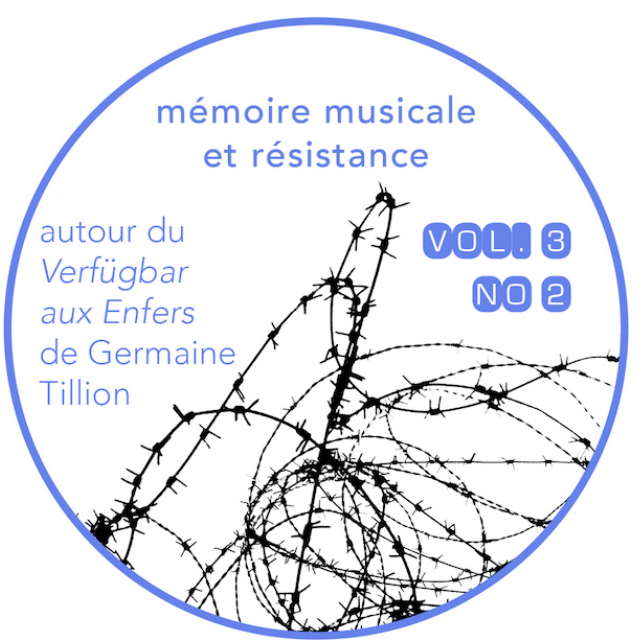
au camp de concentration pour femmes de Ravensbrück. " Euvre » de survie conçue de façon collective et faisant appel à un vaste répertoire de mélodies connues dans les genres les plus divers, Le Verfügbar aux Enfers est un cas exemplaire permettant d'éclairer les interactions complexes entre musique, chant, mémoire et résistance dans les camps pendant la Seconde Guerre mondiale et au-delà.

C'est là l'objet même du projet d'équipe de recherche interdisciplinaire « Mémoire musicale et résistance dans les camps ", codirigée par les directeurs invités de ce numéro, et dont est issue la présente publication. Les travaux de cette équipe ont notamment donné lieu, à l'automne 2014, à un séminaire interdisciplinaire éponyme, proposé par Marie-Hélène Benoit-Otis et Philippe Despoix à l'Université de Montréal. Plusieurs des textes rassemblés ici ont d'abord été exposés dans le cadre de ce séminaire, 
que ce soit sous la forme de conférences invitées ou de travaux de recherche réalisés par les étudiants. Ce dossier réunit donc à la fois des interventions de notre équipe de recherche, de spécialistes invités et d'étudiants-chercheurs, dans une imbrication que nous avons souhaitée aussi étroite que possible.

Après l'article introductif de Philippe Despoix et Marie-Hélène Benoit-Otis présentant les grandes lignes de notre approche analytique du Verfügbar aux Enfers comme un document de conception orale, Djemaa Maazouzi trace un portrait intellectuel inédit de Germaine Tillion, qui souligne le lien fondamental entre réflexion et action solidaire caractérisant son parcours dès cette singulière opéretterevue. Pascal Blanchet et Ariane Santerre explorent de leur côté le réseau de citations aussi bien musicales que littéraires - dont est constitué le document de Tillion : le premier s'intéresse spécifiquement aux figures féminines des opérettes des $\mathrm{XIX}^{\mathrm{e}}$ et $\mathrm{XX}^{\mathrm{e}}$ siècles dont on retrouve des échos dans Le Verfügbar aux Enfers, et la seconde retrace de manière complémentaire et comparée le réseau d'intertextualité littéraire dans lequel s'inscrit ce texte. Cécile Quesney propose ensuite un tour d'horizon de la réception de ce document depuis sa publication en 2005, que ce soit du côté des études publiées jusqu'à ce jour ou de celui des déjà nombreuses mises en scène réalisées ces dernières années. Christophe Gauthier, quant à lui, s'interroge sur les possibilités de réalisation musicale de l'opérette-revue conçue dans le dénuement du camp, et propose une reconstruction originale de l'un de ses numéros chantés. Cette perspective musicale est complétée par Catherine Harrison-Boisvert et Caroline MarcouxGendron, dont l'article explore les enjeux de l'interprétation vocale du Verfügbar aux Enfers aussi bien du point de vue musicologique que de celui de leur pratique du chant. Enfin, dans un post-scriptum abordant sous un angle cinématographique le potentiel de résistance de la musique, Chloé Huvet analyse la façon dont le personnage principal du film Le pianiste de Roman Polanski mobilise sa compétence musicale comme un moyen de survivre.

Le numéro est complété par un ensemble de comptes rendus permettant d'élargir la réflexion en abordant des thématiques connexes, tels les liens entre humour et Shoah (Rire, mémoire, Shoah recensé par Gabriel Paquin-Buki), la création théâtrale dans les camps (Performing Captivity, Performing Escape par Jean-Philippe Michaud) ou la résilience par la musique chez la pianiste déportée Alice Herz-Sommer (le film The Lady in Number 6 par Laurence Manning). Catherine Harrison-Boisvert recense enfin une publication toute récente et en prise directe avec notre dossier : le catalogue de l'exposition Les armes de l'esprit, Germaine Tillion 1939-1954 présentée en 2015 par le Musée de la Résistance et de la Déportation de Besançon. 
ARTICLES

Le Verfügbar aux Enfers - un document de conception orale. Ou comment résister par le rire à l'horreur des camps

1 Philippe Despoix et Marie-Hélène Benoit-Otis

Germaine Tillion. Credo du « solidus " et sympathie à la lettre

22 Djemaa Maazouzi

Chants de femmes triomphantes. Les opérettes citées dans Le Verfügbar aux Enfers 38 Pascal Blanchet

L'intertextualité dans Le Verfügbar aux Enfers et d'autres témoignages concentrationnaires. Une comparaison entre les périodes d'incarcération et d'après-guerre

55 Ariane Santerre

Le Verfügbar aux Enfers. Études et mises en scène contemporaines

78 Cécile Quesney

Reconstruire les numéros chantés du Verfügbar aux Enfers. Le cas de 1'air de Rosine

99 Christophe Gauthier

L'interprétation vocale du Verfügbar aux Enfers au $\mathrm{XXI}^{\mathrm{e}}$ siècle

117 Catherine Harrison-Boisvert et Caroline Marcoux-Gendron

Le pianiste de Roman Polanski (2002). Survivre et exister par la musique

135 Chloé Huvet

NOTES DE TERRAIN

Rire, mémoire, Shoah, d'Andréa Lauterwein (dir.)

157 Gabriel Paquin-Buki

Performing Captivity, Performing Escape. Cabarets and Plays from the Terezin/ Theresienstadt Ghetto, de Lisa Peschel

165 Jean-Philippe Michaud

The Lady in Number 6. Music Saved My Life, court-métrage documentaire de Malcolm Clarke

170 Laurence Manning

Les armes de l'esprit, Germaine Tillion 1939-1954, catalogue de l'exposition présentée par le Musée de la Résistance et de la Déportation de Besançon, 26 mai20 septembre 2015, Citadelle de Besançon

176 Catherine Harrison-Boisvert 
Analyses et interprétations de la musique, la mélodie du berger dans le Tristan et Isolde de Richard Wagner, de Jean-Jacques Nattiez

183 Claude Abromont

Couverture : image issue de Pixabay, sous licence Domaine public.

Graphisme : Solenn Hellégouarch. 